\title{
RUPPELT, Georg, SOLF, Sabine, Lexikon zur Geschichte und Gegenwart der Herzog-August-Bibliothek Wolfenbüttel
}

Joseph Morsel

\section{OpenEdition}

Journals

Édition électronique

URL : http://journals.openedition.org/ifha/1548

DOI : $10.4000 /$ ifha. 1548

ISSN : 2198-8943

Éditeur

IFRA - Institut franco-allemand (sciences historiques et sociales)

Référence électronique

Joseph Morsel, « RUPPELT, Georg, SOLF, Sabine, Lexikon zur Geschichte und Gegenwart der HerzogAugust-Bibliothek Wolfenbüttel », Revue de l'IFHA [En ligne], Date de recension, mis en ligne le 01 janvier 1996, consulté le 22 septembre 2020. URL : http://journals.openedition.org/ifha/1548 ; DOI : https:// doi.org/10.4000/ifha.1548

Ce document a été généré automatiquement le 22 septembre 2020.

(C)IFHA 


\title{
RUPPELT, Georg, SOLF, Sabine, Lexikon zur Geschichte und Gegenwart der Herzog-August-Bibliothek Wolfenbüttel
}

\author{
Joseph Morsel
}

Il n'est sans doute pas inutile de signaler cette présentation du paysage des bibliothèques françaises, explicitement destiné aux étudiants, enseignants et chercheurs allemands en "romanistique« (langues et cultures romanes). Bien que soit parue en 1988-1989 une histoire des bibliothèques françaises jusqu'à la Révolution et que les vifs débats autour de la Bibliothèque de France se soient traduits par une multiplication de publications en France, cet ouvrage présente deux avantages. La position des deux auteurs en dehors du débat sur la légitimité ou non d'une Bibliothèque de France dans un paysage de bibliothèques que beaucoup considèrent comme sinistré permet de disposer ici d'une vue d'ensemble à peu près impartiale et à visée plutôt informative. Sont en outre signalés en bibliographie des titres et revues le plus souvent inconnus aux utilisateurs des bibliothèques, mais où sont présentées des données non inutiles pour comprendre les enjeux des débats en question. Par ailleurs, l'orientation vers un public étranger et entre autres étudiant l'amène à considérer de manière non allusive la situation des bibliothèques en France, leur histoire (qui constitue une première partie, allant de l'Antiquité à 1945), l'organisation bibliothéconomique du pays (formation et organisation des bibliothécaires, services transrégionaux et nationaux de prêt et de catalogage, actions d'institutions publiques à ce niveau: deuxième partie), les différents types de bibliothèques et leur évolution récente (depuis 1945, y compris ce que l'on savait en 1990 de la Bibliothèque de France, ainsi que quelques réseaux de bibliothèques privées: parties 3 à 7) et enfin quelques aperçus sur la distribution commerciale des livres. Une riche bibliographie française et allemande, une liste d'adresses importantes en la matière et un index complètent cet utile panorama. 
2 Certaines bibliothèques françaises seront certainement concernées par une nouvelle collection, consacrée aux grandes bibliothèques européennes, dont le lexique historique et pratique consacré à la Bibliothèque Herzog-August de Wolfenbüttel, en Basse-Saxe, est le premier volume - et le premier du genre. Plus d'une centaine de collaborateurs ont œuvré sur les 365 rubriques qui le composent et présentent l'histoire de la bibliothèque (cf. par ex. les rubriques »August der Junge« ou encore »Lessing, Gotthold Ephraim«), ses fonds (par exemple »Helmstedter Handschriften«), les types d'ouvrages (»Fabelbücher«, "Kochbücher«, etc.), certaines pièces remarquables (»Evangeliar Heinrichs des Löwen«, „Sachsenspiegel«), les services proposés (»Bücherauto«, »Fotowerkstatt« »Restaurant« et autres), les divers bâtiments de la Bibliothèque. Un projet original. 